\title{
MYOCARDIAL PERFUSION SCINTIGRAPHY AND DUKE'S TREADMILL SCORE WITH INVASIVE ANGIOGRAM: A COMPARATIVE STUDY IN PATIENTS
}

\author{
Harish Basavaraja1, Sowmya Horatti Eshwarappa ${ }^{2}$
}

${ }^{1}$ Assistant Professor, Department of Nuclear Medicine, Kidwai Institute of Oncology, Bangalore.

${ }^{2}$ Assistant Surgeon, Department of Nuclear Medicine, Kidwai Institute of Oncology, Bangalore.

\begin{abstract}
Coronary Artery Disease (CAD) is the leading cause of death and disability of mankind. There are various screening and diagnostic tests available for evaluating CAD. We tried to evaluate Duke's treadmill score and Myocardial perfusion scan for evaluating CAD with invasive coronary angiogram as gold standard; 130 patients were included in the study. All underwent treadmill test and MPS. In results it was found that patients with High (7\%) and Low (32\%) Duke's score, MPS did not add much incremental value. But majority of the patients fell in moderate Duke's score (60\%). MPS played a major role in this moderate Duke's score, which sub-classified it into Normal (64\%), Mild (22\%) and Severe (7.7\%). In addition, correlation of MPS with invasive angiogram was very good with sensitivity of $85-90 \%$ and specificity of $87-100 \%$. MPS plays important role in evaluating $\mathrm{CAD}$, in that it provides incremental value in patients with moderate Duke's treadmill score.
\end{abstract}

\section{KEYWORDS}

Duke's Treadmill Score, Myocardial Perfusion Scintigraphy.

HOW TO CITE THIS ARTICLE: Basavaraja H, Eshwarappa SH. Myocardial perfusion scintigraphy and Duke's treadmill score with invasive angiogram: a comparative study in patients. J. Evolution Med. Dent. Sci. 2016;5(70):5062-5066, DOI: $10.14260 /$ jemds/2016/1150

\section{INTRODUCTION}

Coronary Artery Disease (CAD) is the leading cause of death and disability in both developed and developing countries. Proper risk stratification is critical for the management of the patient with known or suspected CAD. Decisions regarding revascularisation procedure or medical therapy can only be made after accurately identifying the patients who may benefit with the given treatment strategy.

The fact that most human die of Ischaemic Heart Disease (IHD) than any other disease demands that better methods be developed to diagnose IHD and to determine response to therapy.(1) World Health Task Force on Nomenclature defines Myocardial Ischaemia as "Diminished supply of blood in respect to cellular demands caused by coronary perfusion changes."(2) This definition is used to describe any patient with IHD caused by CAD with all other possible causes of ischaemia placed in other category.

The detection of myocardial ischaemia relies on measurements at the cellular level, because it is at this level that energy and cellular needs meet. In the myocardium, increased oxygen demand should be met by almost entirely by increased coronary flow. Hence, myocardial function and myocardial blood flow are tightly coupled.(3,4) Ischaemia is a highly dynamic process because of its heavy dependence on oxidative metabolism. The contractile function of myocardial cells become impaired during the ischaemic insult lasting for 1-2 secs., because anaerobic metabolism cannot adequately satisfy the metabolic demands of the myocardial cells.(5) The detrimental effects of ischaemia as short as 20-30 mins., lead to necrosis.

Financial or Other, Competing Interest: None.

Submission 23-07-2016, Peer Review 18-08-2016,

Acceptance 23-08-2016, Published 31-08-2016.

Corresponding Author:

Dr. Harish Basavaraja,

No. 8, Shankari Krupa,

I Stage, II Cross, KHB Colony,

Basaveswaranagara,

Bangalore-560079.

E-mail: reachharishb@gmail.com

DOI: $10.14260 /$ jemds $/ 2016 / 1150$
Most of the myocardial cell's energy utilisation goes to maintain the contractile state. If energy is not constantly replenished by oxidative metabolism, energy stores fall, metabolic by-products accumulate and contractile activity declines.(6) Therefore, direct serial tissue measurements of myocardial energy stores (creatine phosphate and adenosine triphosphate) can provide a sensitive guide to the presence of absence or ischaemia,(7) although technically this is extremely difficult. Other less direct metabolic measurements of ischaemia include local $\mathrm{PCO}_{2}$, lactate production, lactate pyruvate ratio, potassium or phosphate release. However, all these measurements require myocardial sampling and can be used only in experimental animals. Measurements of coronary sinus blood require cardiac catheterisation, and these measurements can be altered by conditions other than IHD.

The objective demonstration of ischaemia by Echocardiogram (ECG) or Myocardial Perfusion Scintigraphy (MPS) in patients with $\mathrm{CAD}^{(8,9)}$ is associated with a significantly higher morbidity and mortality. The cellular basis of ischaemia is represented in Table I. Invasive and noninvasive techniques currently available for evaluating patients with CAD is outlined.

\begin{tabular}{|c|c|}
\hline Pathophysiological Changes & Diagnostic Tests \\
\hline Supply-demand imbalance & Myocardial Perfusion Scintigraphy (MPS) \\
\hline Abnormal Wall motion & $\begin{array}{l}\text { Radionuclide ventriculogram (MUGA) } \\
\text { Gated MPS } \\
\text { ECHO }\end{array}$ \\
\hline LV diastolic dysfunction & $\begin{array}{l}\text { Radionuclide ventriculogram (MUGA) } \\
\text { Gated MPS } \\
\text { Doppler Echocardiography }\end{array}$ \\
\hline LV systolic dysfunction & $\begin{array}{l}\text { Radionuclide ventriculogram (MUGA) } \\
\text { Gated MPS } \\
\text { Doppler }\end{array}$ \\
\hline Electrophysiologic changes & Exercise ECG (TMT) \\
\hline Clinical expression (Angina) & History and physical examination \\
\hline
\end{tabular}

Table 1: Currently Available Non-Invasive Diagnostic Tests for Diagnosis of Myocardial Ischaemia 
Exercise treadmill testing remains the cornerstone and acts as gatekeeper and decide further management. The pioneering work started by Master(10) has led to an established role for the ECG-monitored exercise stress test in the evaluation of IHD. Few reports questioned the usefulness of exercised ECG, especially in the populations where IHD would be expected to have a low prevalence. Nevertheless, ambulatory exercise ECG testing supplies information useful for evaluating predisposition to ischaemia during daily activity. The test is considered as positive when there is typical angina and $>1 \mathrm{~mm}$ reversible ST depression during a stress test.

\section{Few Drawbacks of Stress ECG are}

- Sensitivity is at range of $49 \%$ to $80 \%$ and the specificity is about $41-95 \%$ (when $1 \mathrm{~mm}$ of ST depression was defined as positive result). These wide ranges decrease the utility of the test in low probability cases for CAD.

- Electrocardiographic changes (ST depression) may not occur until the contractile state is already impaired, which suggests that a certain level of cellular ischaemia must be reached prior to clinically apparent ECG changes.

- The effect of coronary collateral blood supply to the myocardium distal to stenosis and the extent of the vessel disease are difficult to interpret.

- The presence of arterial stenosis do not necessarily imply that a region of myocardial tissue is ischaemic, but rather that there is a potential for the attenuation of regional coronary reserve capacity.

- Other conditions like abnormal resting ECG (LBBB), prior myocardial infarction, drug intake and left ventricle hypertrophy can make interpretation of Stress ECG difficult.

To overcome above drawbacks, many stress scores were derived. However, most of them required calculator and computer for calculation of scores, hence were not applicable for routine practice. However, Duke treadmill score is a simple and composite measure of functional capacity (Duration of exercise) and stress-induced ischaemia (ST-T changes in ECG due to inadequate blood supply). Duke's score in addition is able to risk stratify patients into low, intermediate and high risk group.

Performing a simple treadmill test and deriving the Duke's score classified most of the suspected patients of CAD in the intermediate group. Hence, a better diagnostic test was sought for, which had better sensitivity and specificity. Myocardial Perfusion Scintigraphy (MPS) is one such noninvasive test, which plays a very important role to risk stratify the patients in suspected CAD.

Regional abnormalities in myocardial perfusion occur in continuum from minor relative differences in flow without metabolic or regional functional consequences to full expression of myocardial ischaemia with systolic and diastolic dysfunction. It is based on the occurrence of flow heterogeneity induced during hyperaemic stress in patients with suspected CAD. Hence, the test has higher sensitivity (92\%) and higher specificity (80\%) compared to simple TMT and Duke's score.

Invasive Coronary Angiography (CAG) is at present considered as gold standard for diagnosing CAD. It is based on the extent of luminal narrowing with coronary atherosclerosis.

Luminal narrowing of $>75 \%$ is considered as significant stenosis and revascularisation is sought for. In cases of $<75 \%$ stenosis, the question still remains whether it is haemodynamically significant. Hence, these patients undergo MPS stress-test protocol, and intervention is done only in those patients who have positive MPS.

Hence, all the above three tests TMT (Duke's score), MPS and CAG are interdependent in diagnosis and management of patients with CAD. Bayes' theorem states that reliability or interpreting a less than perfect diagnostic test not only depends on sensitivity and specificity of the test, but also depends on prevalence of the disease. Hence, a clinical judgment is very essential in interpretation of these diagnostic tests.

This study shows comparison of DTS and MPS in risk stratifying the patients with CAD in addition.

\section{AIM AND OBJECTIVES}

1. Primary Objective: To correlate Duke's treadmill score at TMT with MPS findings in defining the global myocardial ischaemia.

2. Secondary Objective: To compare the sensitivity and specificity of Duke's Treadmill score and MPS, taking CAG findings as the gold standard in patients in whom all three tests are done.

\section{MATERIALS AND METHODS}

\section{Inclusion Criteria}

All the patients with known and suspected CAD who underwent MPS and who were able to undergo Bruce Treadmill Test during the period between Jan 2007 to Aug 2007.

\section{Exclusion Criteria}

Patients with known history of Myocardial Infarction and patients with abnormal baseline ECG (RBBB, Conduction block).

\section{Duke's Score}

Duke's Treadmill Score was derived using three parameters.

\section{Exercise Time-5 (ST Shift) - 4 (Angina Severity)}

a. Duration of exercise (In minutes).

b. ST shift in ECG (In mm).

c. Angina severity ( 0 denotes no angina; 1 mild angina; and 2 limiting angina).

Based on the DTS the risk was stratified into three Groups:

\begin{tabular}{|c|c|c|}
\hline & Risk Group & DTS \\
\hline 1. & Low & $>4$ \\
\hline 2. & Moderate & -10 to +4 \\
\hline 3. & High & $<-10$ \\
\hline
\end{tabular}

99mTc-MIBI-stress-rest myocardial perfusion SPECT scintigraphy.

Myocardial SPECT scintigraphy was performed with 99mTc sestamibi using of a single day "stress-rest" protocol according to ASNC guidelines. 
Interpretation of the scan was done semi-quantitatively by visual analysis assisted by circumferential profiles analysis using Entegra software.

Both exercise and resting tomographic views were reviewed side by side by experienced nuclear medicine physician; 17-segment model was used for reporting.

An abnormal study was considered where there were reversible perfusion defects. A reversible perfusion defect was defined as perfusion defects on the exercise images that partially or completely resolved at rest in $>2$ contiguous segments in 17 segment model. Based on the degree of intensity and Summed Stress Score (SSS) reversible perfusion defects were divided into following:

\begin{tabular}{|c|c|c|}
\hline & SSS & Pattern/Degree of Uptake \\
\hline 1 & 0 & Normal \\
\hline 2 & 1 & Mildly reduced \\
\hline 3 & 2 & Moderate-severely reduced \\
\hline
\end{tabular}

\section{Coronary Angiography}

Invasive Coronary Angiography was done under local anaesthesia in the cath lab. Under fluoroscope guidance, a tiny catheter advanced from the patient's groin or arm into the opening of the arteries. A small amount of radiographic contrast material was injected into each artery and X-ray was taken to reveal any blockages and their extent. All the standard views were taken and interpreted by two experienced cardiologists. The report included description of all the three vessels (LAD, LCX, RCA) and major branches with the extent of luminal narrowing expressed in percentages.
For the purposes of statistical analysis and comparison with perfusion imaging, angiographic findings are classified into LAD, RCA and LCx territories.

- $\quad$ LAD and their branches supply anterior wall, septum and apical areas.

- $\quad$ Lateral wall area is supplied by LCX, OM branches.

- Inferior wall area is supplied by RCA, PDA and PLV.

\section{RESULTS}

\section{Study Design}

130 patients were enrolled in the study between Jan 2007 to Aug 2007. A correlation descriptive study was done to know relationship between Duke's treadmill score at TMT with MPS findings for severity of ischaemia and also to correlate ischaemia with extent of stenosis in CAG. Age distribution of patients studied included between 31-80 yrs. (Mean $\pm S D=57.14 \pm 8.12$ ). Sex distribution of patients $M / F$ ratio was 112/18.

\begin{tabular}{|c|c|c|c|}
\hline Duke's Score & Grade & $\begin{array}{c}\text { No. } \\
(\mathbf{n = 1 3 0})\end{array}$ & $\mathbf{\%}$ \\
\hline$>4$ & Low & 42 & 32.3 \\
\hline-10 to 4 & Moderate & 78 & 60.0 \\
\hline$<-10$ & High & 10 & 7.7 \\
\hline \multicolumn{4}{|c|}{ Table 2: Duke's Score } \\
\hline
\end{tabular}

\begin{tabular}{|c|c|c|}
\hline MPS & $\begin{array}{c}\text { No. } \\
\text { (n=130) }\end{array}$ & \% \\
\hline Normal & 88 & 67.7 \\
\hline Mild & 27 & 20.8 \\
\hline Mod-Severe & 15 & 11.5 \\
\hline \multicolumn{2}{|c}{ Table 3: MPS } \\
\hline
\end{tabular}

\begin{tabular}{|c|c|c|c|c|c|}
\hline \multirow{2}{*}{ Duke's Score } & \multirow{2}{*}{ No. of Patients } & \multicolumn{3}{|c|}{ MPS } & \multirow{2}{*}{ P Value } \\
\hline & & Normal & Mild & Mod-Severe & \\
\hline Low & 42 & $37(88.1 \%)$ & $4(9.5 \%)$ & $1(2.4 \%)$ & $0.0016^{* *}$ \\
\hline Moderate & 78 & $50(64.1 \%)$ & $22(28.2 \%)$ & $6(7.7 \%)$ & $0.0157^{*}$ \\
\hline High & 10 & $1(10.0 \%)$ & $1(10.0 \%)$ & $8(80.0 \%)$ & $<0.001^{* *}$ \\
\hline Total & 130 & $88(67.7 \%)$ & $27(20.7 \%)$ & $15(11.5 \%)$ & - \\
\hline \multicolumn{6}{|c|}{ Table 4: Association of Duke's Score with the MPS Score } \\
\hline
\end{tabular}

\section{Table 4 Suggests Following}

- Duke's score (Low) was significantly correlated with the MPS of normal with $88.1 \%$ of patients of Low Duke's score are normal based on MPS.

- Similarly, Duke's score of moderate levels is significantly related to either normal or mild MPS. Together (both Normal and Mild MPS) 92.3\% correlated with Moderate Duke's score.

- High Duke's scores are significantly related to the moderate-severe MPS score with $80.0 \%$ of the patients with high Duke's score had moderate-severe MPS score.

\begin{tabular}{|c|c|c|c|c|c|}
\hline Comparison & $\begin{array}{c}\text { True } \\
\text { +ve }\end{array}$ & $\begin{array}{c}\text { False } \\
\text { +ve }\end{array}$ & $\begin{array}{c}\text { False } \\
\text {-ve }\end{array}$ & $\begin{array}{c}\text { True } \\
\text {-ve }\end{array}$ & Total \\
\hline $\begin{array}{c}\text { MPS vs Angio } \\
\text { (LAD) }\end{array}$ & 9 & 2 & 1 & 14 & 26 \\
\hline $\begin{array}{c}\text { MPS vs Angio } \\
\text { (RCA) }\end{array}$ & 13 & 0 & 2 & 11 & 26 \\
\hline $\begin{array}{c}\text { MPS vs Angio } \\
\text { (LCx) }\end{array}$ & 6 & 1 & 1 & 18 & 26 \\
\hline \multicolumn{7}{|c|}{ Table 5a: Correlation of Abnormal MPS with } \\
Angiogram Findings
\end{tabular}

Example: MPS vs Angio (LAD): 9 are MPS+ and angiogram positive, 2 are MPS+ but angiogram negative, 1 case is MPS negative but angiogram positive; 14 patients both MPS and angiogram normal.

\begin{tabular}{|c|c|c|c|c|c|c|}
\hline Comparison & Sensitivity & Specificity & PPV & NPV & Accuracy & P Value \\
\hline MPS vs Angio (LAD) & 90 & 87.5 & 81.8 & 93.3 & 88.4 & $<0.001^{* *}$ \\
\hline MPS vs Angio (RCA) & 86.6 & 100.0 & 100.0 & 84.6 & 92.3 & $<0.001^{* *}$ \\
\hline MPS vs Angio (LCX) & 85.7 & 94.7 & 85.7 & 94.7 & 92.3 & $<0.001^{* *}$ \\
\hline
\end{tabular}




\begin{tabular}{|c|c|c|c|c|}
\hline Comparison & Normal Angiogram & Abnormal Angiogram & Total & Correlation \\
\hline LAD & 28 & 9 & 37 & $75.6 \%$ \\
\hline RCA & 34 & 3 & 37 & $91.8 \%$ \\
\hline LCX & 28 & 9 & 37 & $75.6 \%$ \\
\hline \multicolumn{2}{|r|}{ Table 6: Correlation of Normal MPS with Angiogram Findings } \\
\hline
\end{tabular}

\section{DISCUSSION}

Duke Treadmill Score, Myocardial Perfusion Scintigraphy and Invasive Coronary Angiography have all demonstrated significant power for diagnosing as well as to assess extent and severity of coronary artery disease in both diagnosed and suspected patients.

Exercise treadmill testing has long been the cornerstone of non-invasive risk stratification. Mark et al, demonstrated that a treadmill score incorporating exercise time, ST segment changes and angina was a powerful predictor of survival.(1,2,3) For outpatients, those with a low-risk DTS had a 99\% 4-y survival compared with a 79\% survival rate for patients with a high-risk score.(11) The DTS maintains prognostic power even in models that incorporate clinical characteristics. ${ }^{(4)}$ Despite the utility of the DTS as a prognostic tool, other investigators have shown that radionuclide imaging techniques offer complementary prognostic information for patient management.

In the present study it is seen that low Duke's score is correlating well with normal MPS, which is further confirmed with CAG which shows either normal non-critical $(<75 \%)$ stenosis in these patients. Moderate Duke's score shows correlation with either normal or mild defect in MPS. This is expected as TMT is a screening test for CAD. Majority of the patient's fall in this intermediate category. Hence, MPS may risk stratify these patients into normal or patients having CAD. However, High Duke's score is correlating with severe defects in MPS suggesting that DTS can delineate patients who has severe CAD.

There is also a good correlation of MPS with CAG findings in majority of the patients. In the present study, all the patients who had angiogram were divided into two categories, viz. who had normal MPS and other who had abnormal MPS (Mild-to-severe defects). It is seen that patients with abnormal MPS correlated very well with CAG findings. Correlation of MPS in all the three-vessel territories in CAG was between $88-93 \%$, sensitivity was as high as 85 $90 \%$ and specificity was as high as $88-100 \%$. However, in the second group, i.e. patients with normal MPS, the correlation with CAG was poorer compared with the previous one. It was between $75-92 \%$. Even though CAG showed $>75 \%$ stenosis MPS was normal, thus haemodynamically significant stenosis can be assessed. In addition good collateralisation from other territories explains the above findings.

In patients who have undergone CABG/Angioplasty, out of 25 patients 5 patients had abnormal MPS suggesting progression of CAD even in grafted or stented vessel. This explains that DTS and MPS can be used to assess progression of CAD even in revascularised patients.

\section{LIMITATIONS OF THE STUDY INCLUDES}

- Number of patients included, especially in high Duke score/severe defects in MPS category.

- Non-availability of angiogram in all patients.

- Since degree of stenosis assessed in CAG is operator dependent, inter-observer variation is unavoidable.
- In case of post CABG patients, angiograms after revascularisation was not available.

\section{CONCLUSION}

Duke Treadmill Score, Myocardial Perfusion Scintigraphy and Coronary Angiogram can be used independently and collectively for diagnosis and prognosis of the patients with Coronary Artery Disease.

Treadmill test is a screening test for CAD. Duke Treadmill score adds incremental value to routine treadmill test by risk stratifying patients into low, intermediate and high risk group. Patients who fall under low and high risk DTS, clinical management decisions can be taken using DTS only has MPS adds only marginal incremental value. But majority of the patients fall in intermediate Duke's score. This is the group where MPS provides predominantly significant clinical impact and management decisions. In the present study, MPS may be considered more sensitive and specific in risk stratifying the patients with CAD, especially intermediate risk group in DTS.

CAG providing anatomy of coronary vessels is considered as gold standard of diagnosing CAD. However, haemodynamic significance of the stenosis need to be assessed at the myocardial level. In present study it shows that MPS findings correlates well with angiographic findings when the cut-off is kept as $>75 \%$ stenosis in their respective territories. It is also seen that few patients with significant stenosis in angiogram showed normal MPS, which can be explained by good collateralisation from other vessels resulting in normal perfusion of the myocardium.

\section{REFERENCES}

1. Nomenclature and criteria for diagnosis of ischemic heart disease. Report of joint international society and federation of cardiology and world health organization task force on standardization of clinical nomenclature. Circulation 1979;59(3):607-9.

2. Messer JV, Wagman RJ, Levine HJ, et al. Patterns of human myocardial oxygen extraction during rest and exercise. J Clin Invest 1962;41:725-42.

3. Braunwald E. Control of myocardial oxygen consumption: physiologic and clinical considerations. Am J Cardiol 1971;27(4):416-32.

4. Braasch W, Gudbjarnason S, Puri PS, et al. Early changes in energy metabolism in the myocardium following acute coronary artery occlusion in anesthetized dogs. Circ Res 1968;23(3):429-38.

5. Wyatt HL, Forrester JS, Tyberg JV, et al. Effect of graded reductions in regional coronary perfusion and total cardiac function. Am J Cradiol 1975;36(2):185-92.

6. Kubler W, Katz AM. Mechanism of early pump failure of the ischemic heart: possible role of adenosine triphosphate depletion and inorganic phosphate accumulation. Am J Cardiol 1977;40(3):467-71.

7. Robb GP, Marks HH. Latent coronary artery disease. Determination of its presence and severity by the exercise electrocardiogram. Am J Cardiol 1964;13(5):603-18. 
8. Goldschlager N, Selzer A, Cohn K. Treadmill stress tests as indications of presence and severity of coronary artery disease. Ann Intern Med 1976;85(3):277-86.

9. Master AM, Jaffe HL. Electrocardiographic changes after exercise in angina pectoris. J Mount Sinai Hosp 1941;7: 629-39.

10. Froelicher VF, Fearon WF, Fergusson CM, et al. Lessons learned from studies of the standard exercise ECG test. Chest 1999;116(5):1442-51.
11. Schuijf JD, Wijns W, Jukema JW, et al. Relationship between non-invasive coronary angiography with multi-slice computed tomography and myocardial perfusion imaging. J Am Coll Cardiol 2006;48(12):250814. 